\title{
Neurophysiological Biomarker of Mild Cognitive Impairment*
}

\author{
Wilfried Dimpfel \\ Justus-Liebig-University Giessen c/o NeuroCode AG, Wetzlar, Germany \\ Email: w.dimpfel@neurocode-ag.com
}

Received 4 March 2014; revised 8 April 2014; accepted 15 April 2014

Copyright (C) 2014 by author and Scientific Research Publishing Inc.

This work is licensed under the Creative Commons Attribution International License (CC BY). http://creativecommons.org/licenses/by/4.0/

(c) (i) Open Access

\section{Abstract}

Mild cognitive impairment is sometimes regarded as related to aging. However, statistically every second case turns into full dementia, which still is resistant to any treatment. It is therefore desirable to recognize deviations from normality as early as possible. This might be feasible by using quantitative EEG analysis in the presence of mental work. The present retrospective data analysis revealed a new quantitative biomarker indicating the degree of impairment. Current source density was calculated from 16 channel EEG using CATEEM ${ }^{\circledR}$ software. Four different conditions were analyzed: relaxed state, performing a d2-concentration test, a calculation performance test and a memory test for 5 min each. Subjects older than 40 years were divided into two groups according to their DemTect score: 13 - $18(\mathrm{HC} ; n=44)$ or 8 - $12(\mathrm{MCI} ; n=45)$. Spectral power was chopped into six frequency ranges (delta, theta, alpha 1 , alpha 2 , beta 1 and beta 2 ). Average spectral power was enhanced in the MCI group in comparison to healthy subjects with respect to delta $(p=0.05)$ during relaxed state when all electrode positions were regarded. With respect to EEG recording during performance of three different psychometric tests it was recognized that mainly spectral changes during performance of the d2-concentration test were related to mild cognitive impairment. With regard to all electrode positions statistically significantly lower spectral power values were reached during the d2-test for delta $(p=0.001)$, theta $(p=0.0001)$ and alpha 1 waves $(p=$ 0.08) in impaired subjects in comparison to healthy subjects. Regarding regions of interest increases of delta and theta power were seen in the fronto-temporal brain during performance of the d2-concentration test. These increases disappeared when looking at MCI data. In the centro-parietal region decreases of alpha and beta 1 power emerged, which were even larger in MCI subjects. No MCI-dependent changes were observed in the other two tests. A correlation was found between psychometric performance of the $d 2$-test and the DemTect score $(r=0.51)$. MCI subjects had statistically significant worse performance in all three mental challenges in comparison to healthy volunteers. It is concluded that MCI can be characterized at an early stage by EEG recording in the relaxed state. High spectral delta and theta power in general and specifically at fronto-

${ }^{*}$ Data was presented as poster at the $6^{\text {th }}$ World Congress on Controversies in Neurology, Vienna, Austria, March 8-11, 2012 and awarded the best one in the category dementia. 
temporal electrode positions (especially at $\mathbf{T}_{3}$ ) was recognized as a biomarker for MCI. A DemTect score of 8-12 was validated as indicative for MCI.

\section{Keywords}

DemTect, Cognition, Psychometry, EEG, Source Density, Mild Cognitive Impairment (MCI), Alzheimer's Disease, CATEEM

\section{Introduction}

Mild cognitive impairment is regarded as a possible transitional stage during the development of Alzheimer's disease [1]. According to literature about every second subject suffering from this impairment develops manifest dementia [2]-[4]. Since progression from mild cognitive impairment to dementia occurs fluently, the problem arises to find a quantitatively defined diagnosis of this stage in order to recognize possible parameters for the risk of development of dementia later on. This early brain dysfunction can be described by considering three different approaches. The first approach consists in using an interactive questionnaire like mini-mental state (MMS [5]) or DemTect [6] [7]. The second approach is represented by use of psychometric tasks (cognitive testing) like the d2-test for concentration, a memory test or performance of arithmetic calculations [8]. The third approach very often is covered by a neurophysiological methodology, usually recording of quantitative electroencephalography [9] [10] or biochemical parameters from cerebrospinal fluid [11]. Thus, a combination of measurements on these three levels should be able to provide a quantitative definition of mild cognitive impairment and fulfil the criteria for an early diagnosis and recognition of the risk potential. Results should also provide the quantitative base for testing of drugs or food supplements aiming at the improvement of these early deficits.

The present investigation deals with the comparison of data from 45 healthy control subjects (HC group; DemTect score 13 - 18) with 44 subjects rated as cognitively impaired on the base of a DemTect score between 8 and 12 (MCI group). Concomitant use of psychometric performance and current source density imaging [12] [13] is used to validate this score range for the diagnosis of mild cognitive impairment as proposed by the DemTect guideline in comparison to being mentally healthy. With respect to EEG, basic conditions (recording during the relaxed eyes open condition) as well as event related EEG during performance of mental tasks [14] are used in order to learn more on possible deficits with respect to special demands. The analysis is based on data from 89 subjects using recordings of the first visit in the laboratory during several clinical studies (EudraCT-Nr.: 2007-004753-29 and EudraCT-Nr. 2009-015827-97).

\section{Methods}

\subsection{Subjects}

Eighty-nine subjects were taken from five consecutive clinical studies. They were asked to perform an interactive questionnaire developed for recognition of mild cognitive impairment (DemTect) before they entered the study consisting of a combined technology of EEG recording in the presence of mental performance of three different psychometric tasks. Volunteers were grouped according to the proposal of the developers of the test and according to the validation results published. Subjects having a score from $8-12$ were assembled into the cognitive impaired group (MCI), those scoring higher from 13 - 18 were taken as healthy control group (HC). Both groups were compared with respect to electric power under the different recording conditions and with respect to psychometric performance.

\subsection{Experimental Procedure}

Subjects (HC group 17 male/27 female, average age 50.5 and 47 years, respectively; MCI group 25 male/20 female, average age 58.1 and 56.6 years, respectively) were sitting alone in a quiet separate room in a comfortable easy chair. The light was dimmed. Baseline recording of 6 min under the condition of eyes open was followed by 
the performance of the d2-test, a mathematical calculation task and a memory test. All experiments took place at the same time of the day (starting at $8 \mathrm{am}$ ).

\subsection{EEG Recording}

The EEG was recorded bipolarly from 17 surface electrodes according to the international 10/20 system [15] against a common average reference calculated from $\mathrm{Cz}$ against all other electrodes as proposed by Lehmann [16] (Computer aided topographical electroencephalometry: CATEEM ${ }^{\circledR}$ from MEWICON CATEEM-Tec GmbH, A-4164 Schwarzenberg, Austria) using an electrocap. EEG recording was performed as reported earlier [17]. Setting was kept constant for each individual throughout the experiment.

\subsection{Current Source Density Analysis}

In this study the EEG was processed not in the potential mode based on voltage [18], but in a surface charge mode obtained by Laplacian estimates also known as current source density analysis (CSD), [12] [13]. Charge is the $2^{\text {nd }}$ derivation of the potential and gives the curvature of the potential curve according to space. Under the condition of using a homogenous, steadily conducting medium surface charge mode provides the source density of the electric flow on the cortex surface. Whereas the EEG in the potential mode tends to produce a more extensive and diffuse picture of changes, Laplacian estimate acts as a spatial filter emphasizing local sources over distant sources (for review see [19]). There is a sharply contrasted display of cortex areas with highly activated generators in the depth of the brain and brain areas with less intensely working generators. Harmony et al. [12] were able to demonstrate, that spectral parameters obtained from the CSD showed higher correlations with computer tomography measures than those calculated from the potential mode of the EEG. We therefore used this methodology in order to describe the focal changes of brain activity.

Brain Imaging was achieved by conversion of numerical values of spectral EEG power into spectral colours and additive colour mixture according to RGB as used in TV settings [20]. Data acquisition and analysis were carried out simultaneously and provide topographical maps displayed on-line on the computer screen. The maps show the relative, time averaged changes of electrical brain activity of each recording condition during mental work in \% of the reference period during relaxation with open eyes.

\subsection{Psychometric Testing}

The $\mathrm{d} 2$ attention test is a well-known standardized validated test. Number of correct answers and number of lines were evaluated as performance index including quality and quantity of answers. Arithmetic test (Concentration Performance Test (CPT) was carried out as described by Düker and Lienert, [8]. Number of solved tasks and correctness gave a performance index. The memory test was applied according to the following schema: a combination of 8 numbers and/or letters (for example: (Dv8L3oPX) was presented on the screen for $4 \mathrm{~s}$. After this no information was given for $10 \mathrm{~s}$. Finally, a fourfold multiple choice including the correct answer was presented for decision. Number of tasks and correctness were evaluated to give a performance index. Each test was presented for 5 minutes. The row of order was kept constant for the sake of direct comparisons of the results under identical conditions.

\subsection{Statistics}

Since EEG data are not normally distributed, the non-parametrical Wilcoxon test was chosen for comparison between the two groups. Data were averaged for each of the recording periods of 5 minutes during eyes open and the different challenges (i.e. in separate for each psychometric test). Statistics gave p values, which are presented at the appropriate site. The absolute power values under the recording condition "eyes open” were taken as reference values and set to $100 \%$ when psychometric tests were performed. Thus, possible physiological changes during test performance are given in \% of these reference values.

In order to differentiate results from healthy subjects and those suffering from mild cognitive impairment data were fed into linear discriminant analysis according to Fischer. Results from the first three discriminant functions were depicted in space ( $\mathrm{x}, \mathrm{y}$ and $\mathrm{z}$ coordinates). Results from the $4^{\text {th }}$ to $6^{\text {th }}$ functions were transformed into colour according to the RGB mode (like in TV). 


\section{Results}

\subsection{Electric Power during the Recording Condition "Eyes Open"}

Comparison of the absolute electric power values with respect to all 17 electrode-positions of the source density EEG of subjects with mild cognitive impairment (MCI; $n=45$ ) with the healthy control group (HC; $n=44)$ revealed massive differences. Higher values of electric power at single electrodes were generally seen in the group of subjects with MCI in comparison to healthy subjects. Global median values regarding all electrode positions were higher in MCI subjects than in HC subjects but did not reach such high statistical significance as with respect to delta (Table 1).

Table 1. Absolute power values for each electrode position under the recording condition of "eyes open” in relaxed position for every frequency range from delta, through theta, alpha 1 , alpha 2, beta 1 and beta 2 . Electrode positions: $\mathrm{C}=$ central, $\mathrm{P}$ = parietal; $\mathrm{F}$ = frontal; $\mathrm{T}$ = temporal; $\mathrm{O}=$ occipital. Even numbers represent the right hemisphere, uneven numbers the left hemisphere. Statistically significant differences with respect to median values regarding all electrodes from healthy subjects (HC; $\mathrm{n}=44$ ) in comparison to individuals with mild cognitive impairment (MCI; $\mathrm{n}=45$ ) are marked by stars before the number. ${ }^{*}=\mathrm{p}<0.05 ;^{* *}=\mathrm{p}<0.01 ;^{* * *}=\mathrm{p}<0.001$.

\begin{tabular}{|c|c|c|c|c|c|c|c|c|c|c|c|c|}
\hline \multicolumn{13}{|c|}{ Eyes Open $\left[\mu V^{2}\right]$} \\
\hline \multirow[t]{2}{*}{ Electrode } & \multicolumn{2}{|c|}{ Delta } & \multicolumn{2}{|c|}{ Theta } & \multicolumn{2}{|c|}{ Alpha 1} & \multicolumn{2}{|c|}{ Alpha 2} & \multicolumn{2}{|c|}{ Beta 1} & \multicolumn{2}{|c|}{ Beta 2} \\
\hline & $\mathrm{HC}$ & MCI & $\mathrm{HC}$ & MCI & $\mathrm{HC}$ & MCI & $\mathrm{HC}$ & MCI & $\mathrm{HC}$ & MCI & $\mathrm{HC}$ & MCI \\
\hline $\mathbf{C z}$ & 2.71 & 3.23 & 0.58 & 0.58 & 0.55 & 0.66 & 0.36 & 0.56 & 0.62 & 0.67 & 0.89 & 1.15 \\
\hline $\mathbf{F z}$ & 3.03 & 3.29 & 0.72 & 0.74 & 0.74 & 0.86 & 0.49 & 0.67 & 0.67 & 0.78 & 0.96 & 1.33 \\
\hline F3 & 2.93 & ${ }^{*} 3.89$ & 0.64 & 0.81 & 0.76 & 0.97 & 0.51 & 0.66 & 1.04 & 1.17 & 2.08 & 2.49 \\
\hline $\mathrm{C} 3$ & 2.10 & *3.65 & 0.52 & 0.56 & 0.58 & 0.77 & 0.63 & 1.04 & 1.24 & 1.40 & 1.76 & 2.04 \\
\hline P3 & 1.71 & ${ }^{*} 2.21$ & 0.42 & 0.40 & 0.52 & 0.63 & 0.48 & 0.65 & 0.88 & 0.72 & 0.77 & 0.63 \\
\hline $\mathbf{P z}$ & 2.49 & 2.48 & 0.54 & 0.53 & 0.58 & 0.75 & 0.52 & 0.69 & 0.67 & 0.70 & 0.66 & 0.62 \\
\hline P4 & 1.73 & 2.17 & 0.35 & 0.42 & 0.44 & 0.55 & 0.44 & 0.73 & 0.76 & 0.71 & 0.73 & 0.74 \\
\hline C4 & 2.36 & 2.89 & 0.50 & 0.55 & 0.57 & 0.78 & 0.70 & 1.04 & 1.39 & 1.45 & 1.96 & 2.31 \\
\hline F4 & 3.52 & 4.12 & 0.72 & 0.78 & 0.86 & 0.91 & 0.62 & 0.82 & 1.11 & 1.33 & 2.60 & 3.75 \\
\hline F7 & 7.07 & 10.59 & 1.39 & 1.41 & 1.38 & 1.79 & 1.15 & 1.42 & 1.98 & 1.87 & 4.75 & 3.16 \\
\hline T3 & 4.16 & 4.62 & 0.88 & 0.89 & 1.22 & 1.31 & 1.23 & 1.32 & 2.44 & 1.78 & 3.36 & 3.10 \\
\hline T5 & 3.19 & 3.79 & 1.10 & 0.96 & 1.74 & 1.51 & 1.37 & 1.25 & 2.07 & 1.70 & 1.84 & 1.34 \\
\hline 01 & 3.10 & ${ }^{* * *} 5.88$ & 0.73 & ${ }^{*} 0.88$ & 0.68 & 1.17 & 0.70 & ${ }^{*} 0.99$ & 1.30 & 1.60 & 1.85 & 2.65 \\
\hline $\mathbf{O 2}$ & 4.00 & ${ }^{* *} 5.46$ & 0.79 & 0.86 & 0.90 & 0.91 & 0.87 & 1.10 & 1.49 & 1.83 & 2.20 & 2.40 \\
\hline T6 & 3.07 & 2.88 & 0.77 & 0.69 & 1.14 & 1.51 & 1.04 & 1.36 & 1.86 & 1.50 & 1.72 & 1.70 \\
\hline $\mathbf{T 4}$ & 3.79 & 4.12 & 0.93 & 0.89 & 1.20 & 1.38 & 1.18 & 1.11 & 2.37 & 2.13 & 3.31 & 3.35 \\
\hline F8 & 7.84 & ${ }^{*} 11.88$ & 1.57 & 1.60 & 1.61 & 1.92 & 1.42 & 1.40 & 2.67 & 2.01 & 6.18 & 3.66 \\
\hline global median & 3.14 & *3.62 & 0.71 & 0.74 & 0.83 & 0.91 & 0.75 & 0.86 & 1.33 & 1.43 & 1.81 & 1.92 \\
\hline
\end{tabular}




\subsection{Spectral Power during Performance of the $\mathrm{d} 2$-Concentration Test}

Regarding the median of all electrode positions during performance of the d2-concentration test statistically different values for the MCI subjects in comparison to healthy control were obtained when recordings in the relaxed state were taken as reference $(100 \%)$. Highest statistical significance for the difference between the healthy and the cognitively impaired group is reached in the delta $(\mathrm{p}<0.001)$ and theta frequency range $(\mathrm{p}<$ $0.001)$. But also lower values for alpha 2 power $(\mathrm{p}<0.08)$ were seen during this challenge. Lower spectral power in the alpha 1 frequency range did not reach statistical significance. No statistically significant differences between MCI subjects and healthy controls were observed with the calculation performance test (CPT) or the memory test (Table 2).

During performance of the d2-test healthy volunteers (HC-group) were able to increase fronto-temporal delta and theta power taking the recording condition "eyes open" as reference (100\%). Highest statistical significance was reached by increases of theta power in frontal and temporal areas of the brain as documented in Figure 1 by statistical analysis for each location in separate. At the same time attenuation of electric power were observed with respect to alpha waves mainly in central areas of the brain.
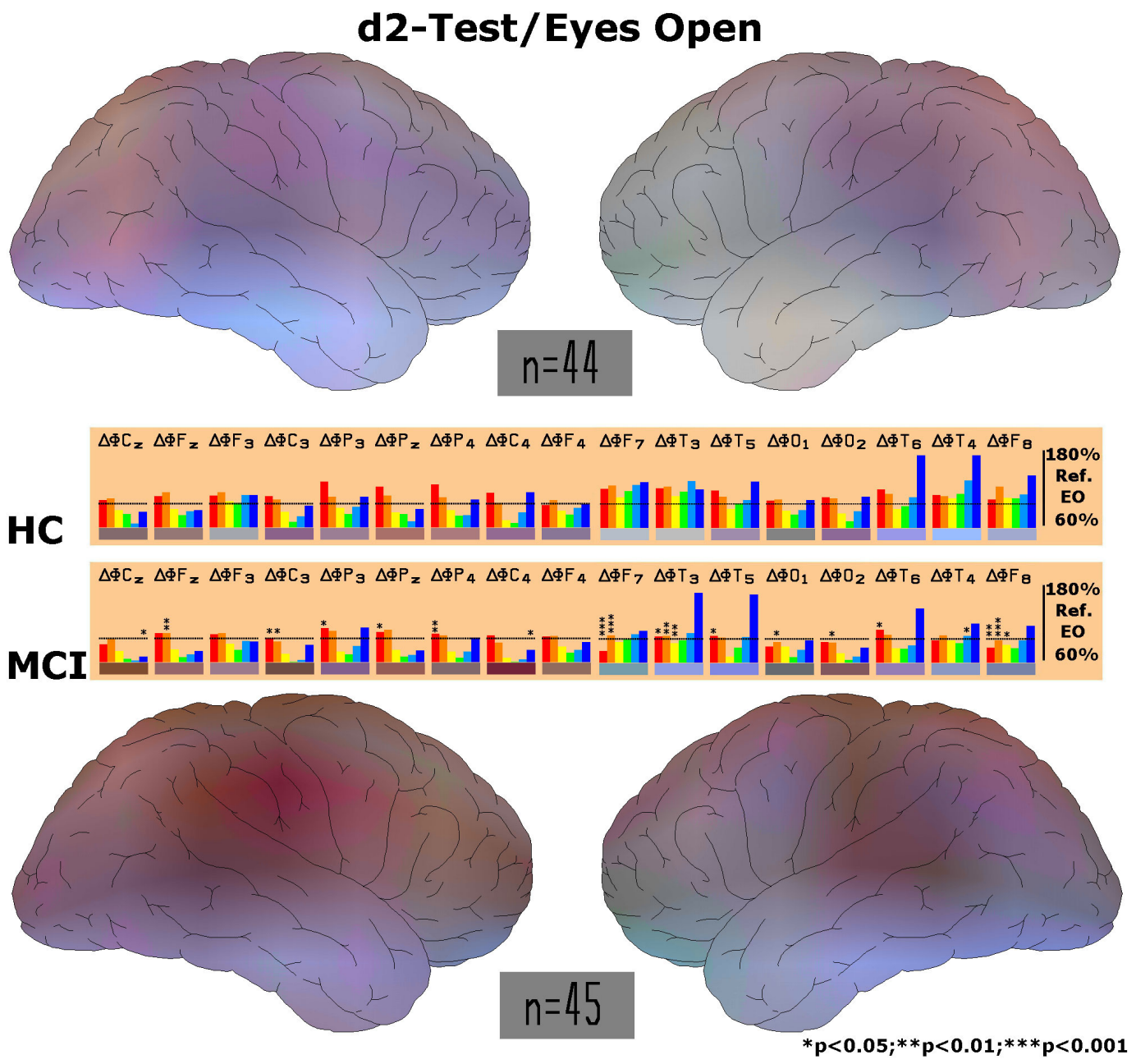

Figure 1. Documentation of statistically significant differences in healthy subjects in comparison to subjects suffering from MCI with respect to every single electrode position under the recording condition of the d2-test. Statistically significant differences between HC and MCI are documented by stars. Brain maps were constructed using the recording condition eyes open as reference. Differences are shown between healthy subjects (HC) and subjects with mild cognitive impairment (MCI) during performance of the d2-test. Please note fronto-temporal brightening in the left hemisphere (right side of upper map) in healthy people compared to lack of such feature in mild cognitive impairment (lower map). 
Opposite to this, subjects diagnosed to have MCI according to "DemTect" score produced significant less electric power regarding delta and theta frequencies during performance of this test. Differences in changes of power with respect to single electrode locations are depicted in Figure 2 for the HC control group (upper panel) and the MCI group (lower panel), respectively. Statistical significance is given in the lower part for each location of recording.

During the course of brain research it has become obvious that electric activity depends on the region where one looks at. Different mental challenges have been recognized to induce quite different patterns of electric activity. This is also seen during performance of a concentration test (Figure 1). Under this recording condition eminent higher delta spectral power values are observed at parietal areas (electrode positions $\mathrm{P}_{3,4}$ ) as well as delta and theta spectral power in fronto-temporal areas represented by the electrode positions $F_{7,8, z}$ and $T_{3,4}$. In MCI subjects these increases disappear nearly completely in a highly statistically significant manner. In addition, alpha waves are depressed in the parietal region during the d2test. This decrease became even more pronounced in mentally impaired subjects. This difference also becomes visible when looking at the electric maps
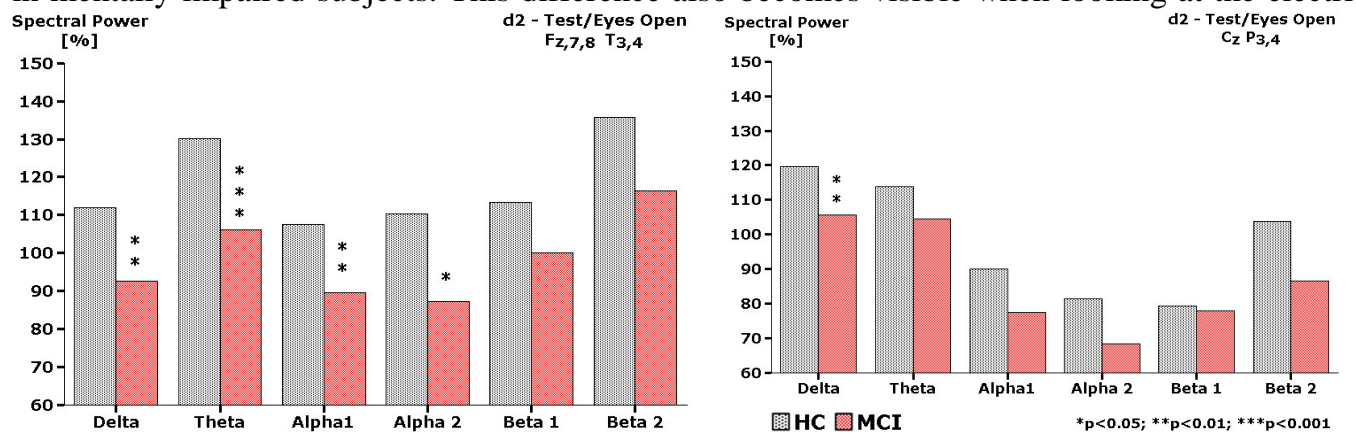

Figure 2. Differences in spectral EEG power between healthy subjects (HC) and subjects suffering from mild cognitive impairment (MCI) with respect to two regions of interest (ROI) when performing the d2-concentration test. Data from the relaxed state are taken as reference. Fronto-temporal region is represented by the electrode positions $\mathrm{F}_{\mathrm{z}, 7,8}$ and centro-parietal region is represented by the positions $\mathrm{C}_{\mathrm{z}}$ and $\mathrm{P}_{3,4}$.

Table 2. Differences of frequency changes (test condition in \% relative to eyes open condition) between healthy subjects (HC) and the MCI group are given for each psychometric test as median values taking all electrode positions in consideration. Statistical significance is given as p-values for the comparison between healthy people (HC) and subjects suffering from mild cognitive impairment (MCI).

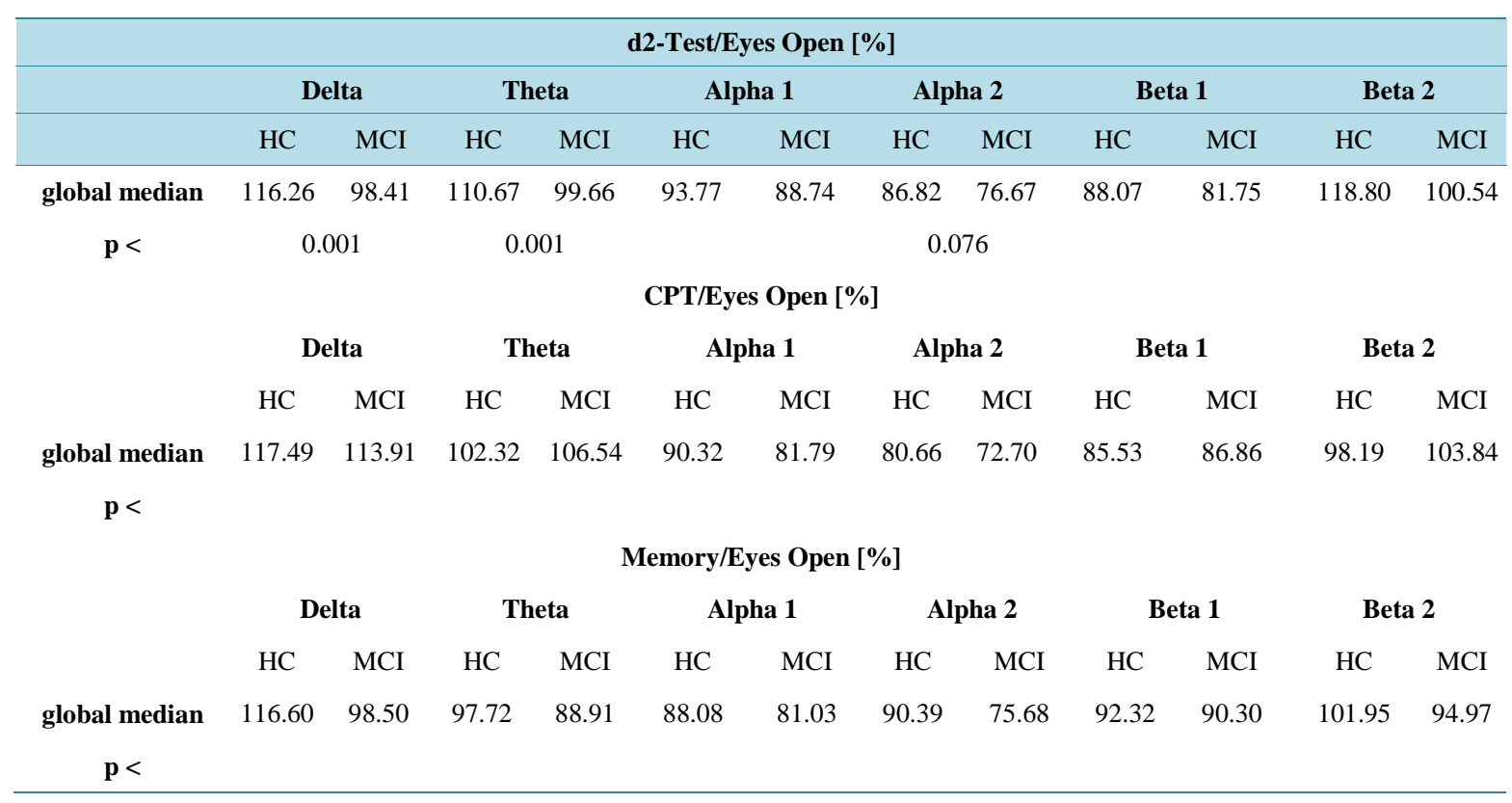

calculated for both groups (Figure 1). Due to the absence of theta power a large difference is seen in fronto- 
temporal areas. Thus, under the recording condition “d2-concentration test” massive differences with respect to spectral power in fronto-temporal and parietal brain regions are visible. The numerical values for these two regions of interest are given in separate as median of the respective fronto-temporal and parietal electrode positions (Figure 2).

\subsection{Electric Power during the Recording Condition "CPT"}

During performance of the calculation-performance-test (CPT) healthy volunteers were able to increase frontal delta and theta power in comparison to the recording condition "eyes open" (set to 100\%). As depicted in the middle bar chart of Figure 3, electrode locations $\mathrm{F}_{7}, \mathrm{~F}_{8}, \mathrm{~T}_{5}$ and $\mathrm{T}_{6}$ show increases of delta and theta power. With respect to this hardly a difference was observed in cognitively impaired volunteers. However, when looking at the brain map depicted in Figure 3, left frontal increases of slow power were attenuated in mildly impaired subjects. Despite the impression of lower production of delta and theta waves in the impaired group (as also documented in Figure 4) this difference was not statistically significant. The same is true for beta power. Global
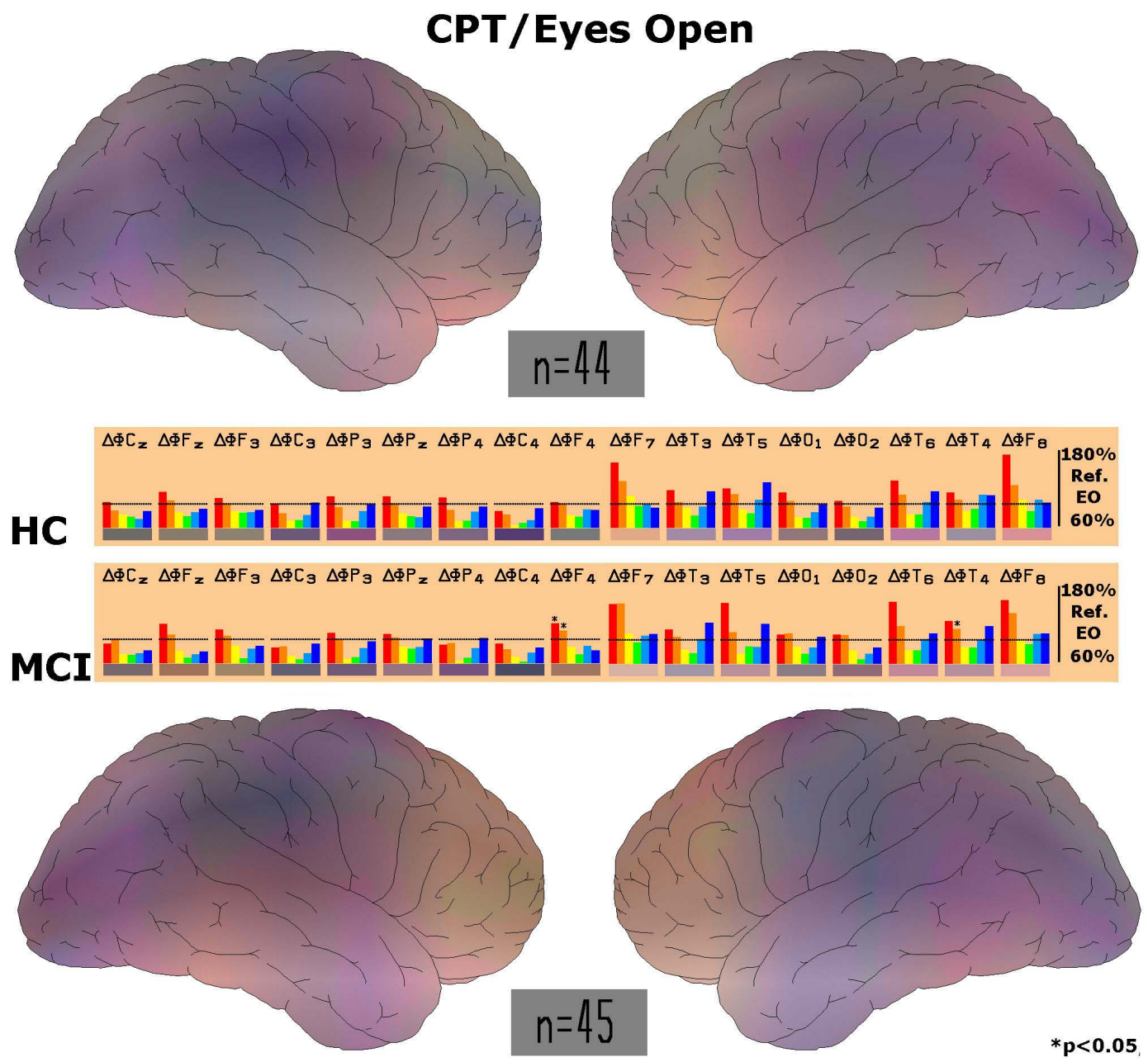

Figure 3. Documentation of statistically significant differences in healthy subjects in comparison to subjects suffering from MCI with respect to every single electrode position under the recording condition of the calculation performance test (CPT). Statistically, significant differences between HC and MCI are documented by a star. Brain maps constructed using the recording condition eyes open as reference. Differences are shown between healthy subjects (HC) and subjects with mild cognitive impairment (MCI) during performance of the d2-test. Please note that only marginal differences between healthy people subjects suffering from mild cognitive impairment are seen. 

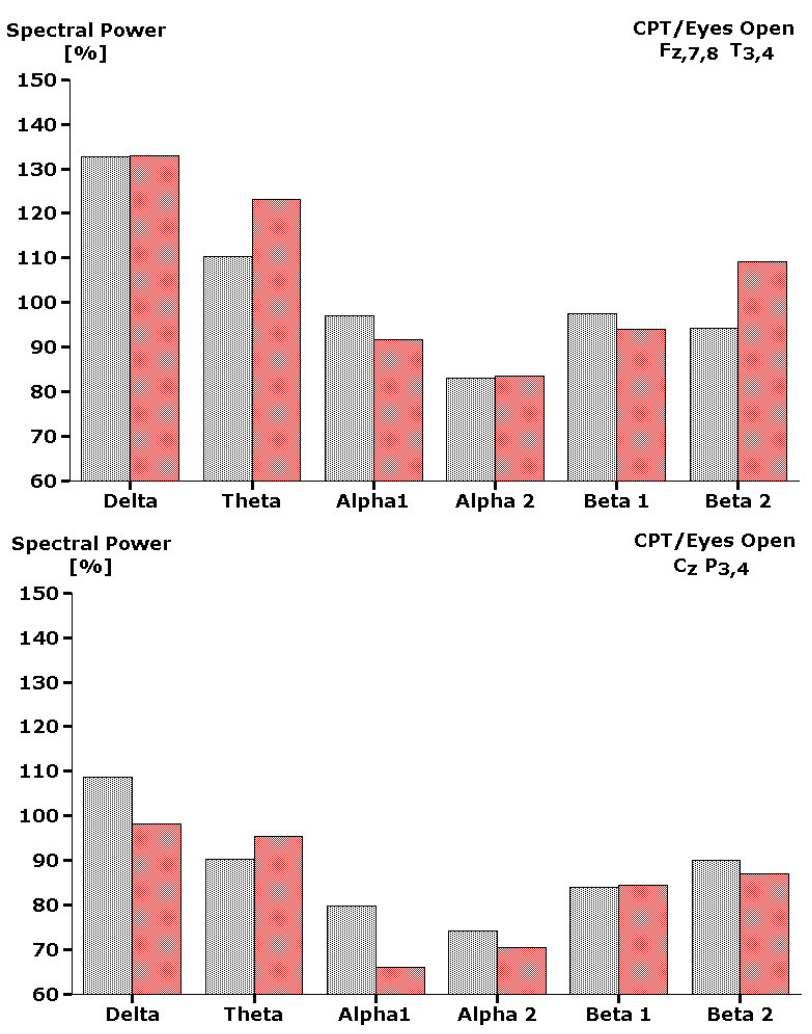

眻HC

Figure 4. Differences in spectral EEG power between healthy subjects (HC) and subjects suffering from mild cognitive impairment (MCI) with respect to two regions of interest (ROI) when performing the calculation performance test. Data from the relaxed state are taken as reference. Fronto-temporal region is represented by the electrode positions $F_{z, 7,8}$ and centro-parietal region is represented by the positions $\mathrm{C}_{\mathrm{z}}$ and $\mathrm{P}_{3,4}$.

median values of electric power with regard to all electrode positions during the performance of the calculation test are given in Table 2 (middle part). Despite some further reduction of alpha 1 and alpha 2 power in mildly impaired subjects this difference in comparison to healthy subjects did not become statistically significant.

This result is underlined by the spectral changes as observed in the two regions of interest. Despite some lower value with respect to alpha 1 power in mildly impaired subjects, the difference did not become statistically significant as depicted in Figure 4.

\subsection{Electric Power during the Recording Condition "Memory Test"}

During performance of the memory test, healthy volunteers were able to increase frontal delta power $\left(\mathrm{F}_{7,8}\right)$ and to some extent also theta power in comparison to the recording condition "eyes open” (set to 100\% in Figure 5). A similar feature was observed in cognitively impaired volunteers. But with respect to delta and theta spectral power cognitively impaired subjects produced somewhat less spectral power (bar chart in the middle of Figure 5). Electric maps in mildly impaired subjects reveal less red colour in the frontal brain due to some but statistically not significant delta decreases. Differences between healthy controls and impaired subjects are also obvious from the regions of interest in Figure 6. Obviously, the lower spectral delta power is statistically not significantly different between the two groups. However, alpha 2 spectral power was significantly less in impaired subjects. When regarding global median values (calculated from all electrode positions) also a tendency of attenuation of alpha 2 spectral power emerged, but which did not become statistically significant at this global measurement (Table 2). 


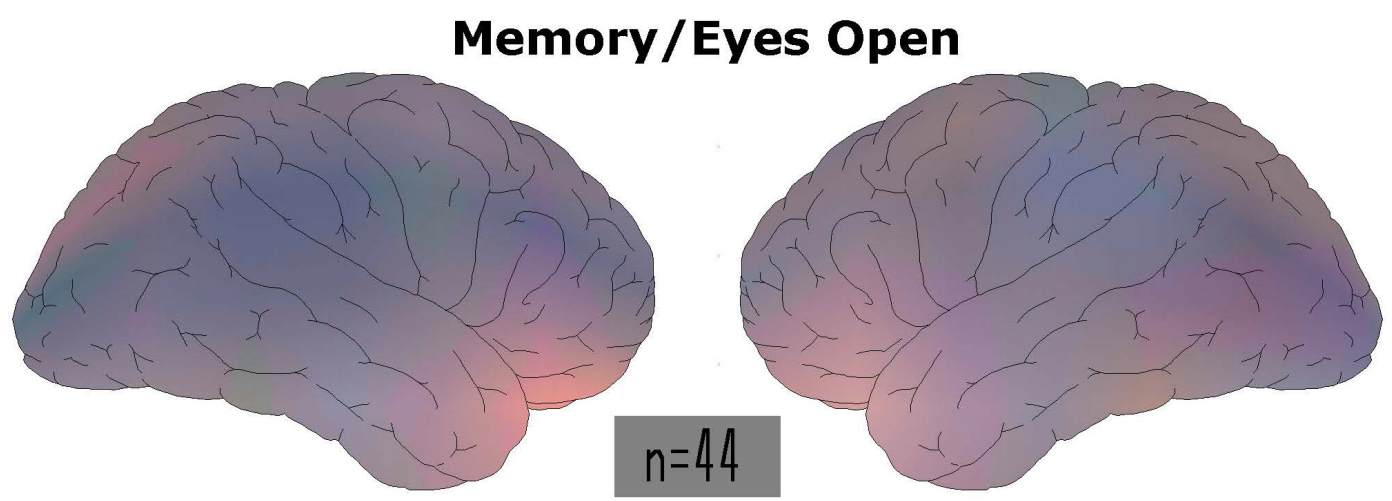

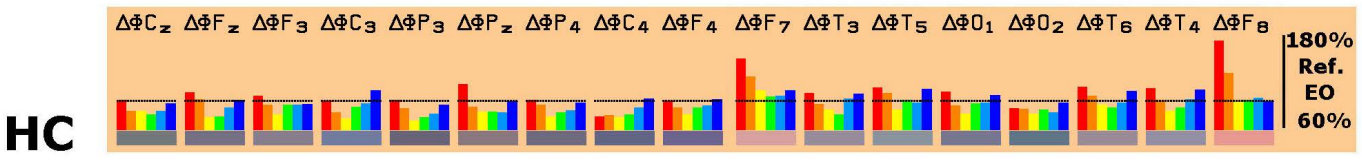

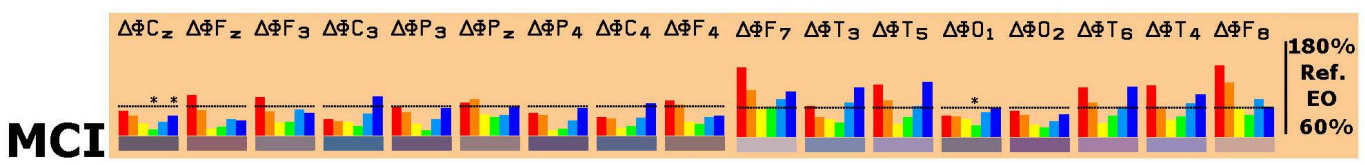

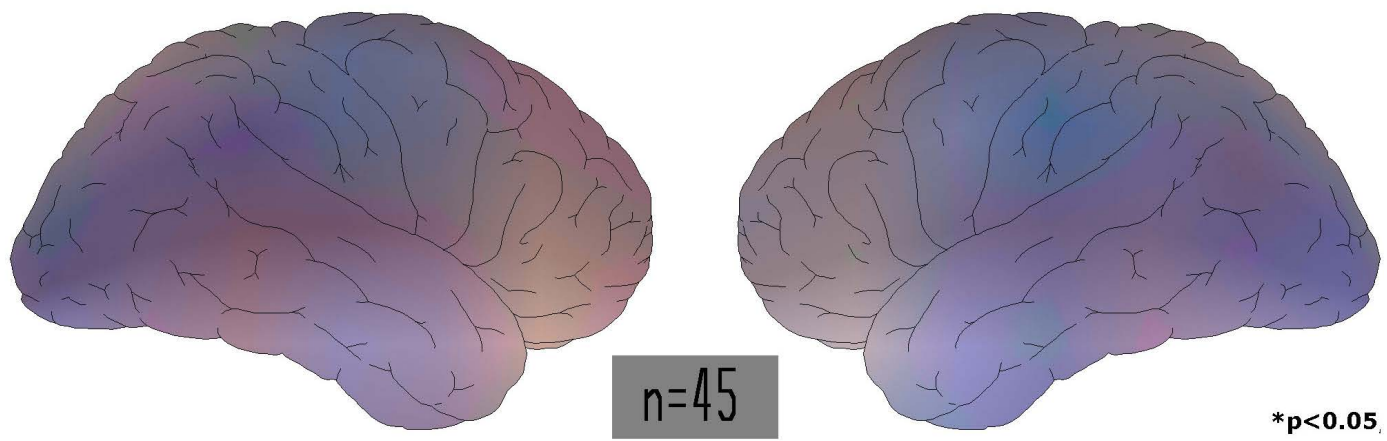

Figure 5. Documentation of statistically significant differences between healthy subjects and subjects suffering from MCI with respect to every single electrode position. Statistically significant differences between HC and MCI subjects are documented by stars. Brain maps are constructed using the recording condition eyes open as reference (100\%). Differences are shown between healthy subjects (HC) and subjects with mild cognitive impairment (MCI) during performance of the memory test. Please note only marginal differences between healthy people and subjects suffering from mild cognitive impairment.
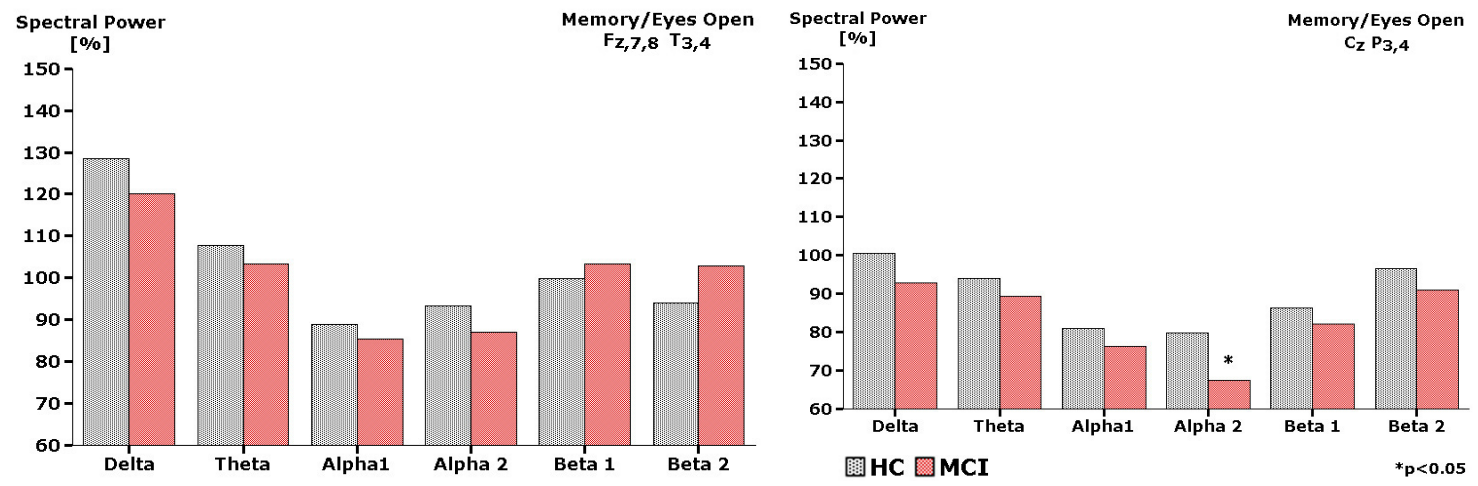

Figure 6. Differences in spectral EEG power between healthy subjects (HC) and subjects suffering from mild cognitive impairment (MCI) with respect to two regions of interest (ROI) when performing the memory test. Data from the relaxed state are taken as reference $(100 \%)$. The frontotemporal region is represented by the electrode positions $F_{z, 7,8}$ and the centroparietal region is represented by the positions $C_{z}$ and $P_{3,4}$. 


\subsection{Comparison by Means of Discriminant Analysis}

Discriminant analysis is a mathematical tool, which allows statistical evaluation of a large set of parameters. Since this quantitative EEG analysis consists of 102 parameters (17 electrode positions $\times 6$ frequency bands) absolute spectral power data from all participants of the studies were fed into this type of analysis. As is documented in Figure 7 healthy controls and impaired subjects can be discriminated from each other with respect to all 4 recording conditions. It is also obvious that the different recording conditions lead to different types of electric brain states. For example the state of "eyes open" can easily discriminated from the state during performance of these psychometric tests.

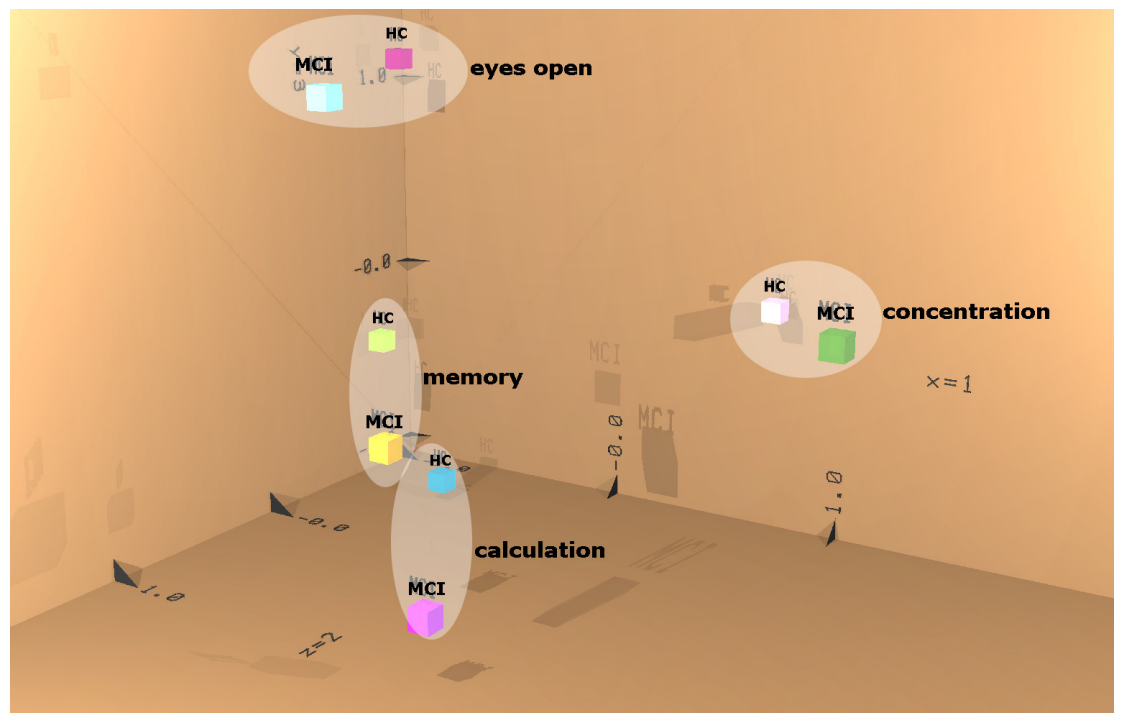

Figure 7. Documentation of results of discriminant analysis for both groups of subjects ( $\mathrm{HC}=$ healthy controls; $\mathrm{MCI}=$ mild cognitive impairment) with respect to performance of psychometric tests and the recording condition relaxed state with "eyes open". Results from the first three discriminant functions are depicted in space (x,y and $\mathrm{z}$ coordinates). Results from the $4^{\text {th }}$ to $6^{\text {th }}$ discriminant functions are depicted using the RGB mode (additive colour mixture of red, green and blue for the result of each function). Single test conditions are marked by arbitrarily brightening.

\subsection{Evaluation of Psychometric Results}

Psychometric performance was documented for each of the three mental tests according to the definition given under "Material and Methods". As described in Table 3 performance of cognitively impaired subjects was always significantly lower in all three tests with respect to an index calculated on the base of quality and quantity of the answers.

In addition, a statistically significant correlation was observed between the psychometric performance during these tests and the score of the "DemTect". These data confirm the cognitive impairment as indicated by the "DemTect" score, since a correlation was observed between the DemTect score and these psychometric results $(\mathrm{r}=0.53 ; \mathrm{p}=0.000001)$ as depicted in Figure 8.

Table 3. Result for psychometric testing (details under material and methods). Mean values and deviation are given besides the statistical significance ( $p$-values) on the left side.

\begin{tabular}{cccccc}
\hline & Mean HC & SEM HC & Mean MCI & SEM MCI & Statistical Significance \\
\hline d2-test & 11.20 & 0.48 & 8.21 & 0.43 & $\mathrm{p}<0.0001$ \\
CPT & 4.46 & 0.59 & 2.93 & 0.49 & $\mathrm{p}<0.03$ \\
Memory & 10.38 & 0.49 & 8.36 & 0.64 & $\mathrm{p}<0.02$ \\
\hline
\end{tabular}




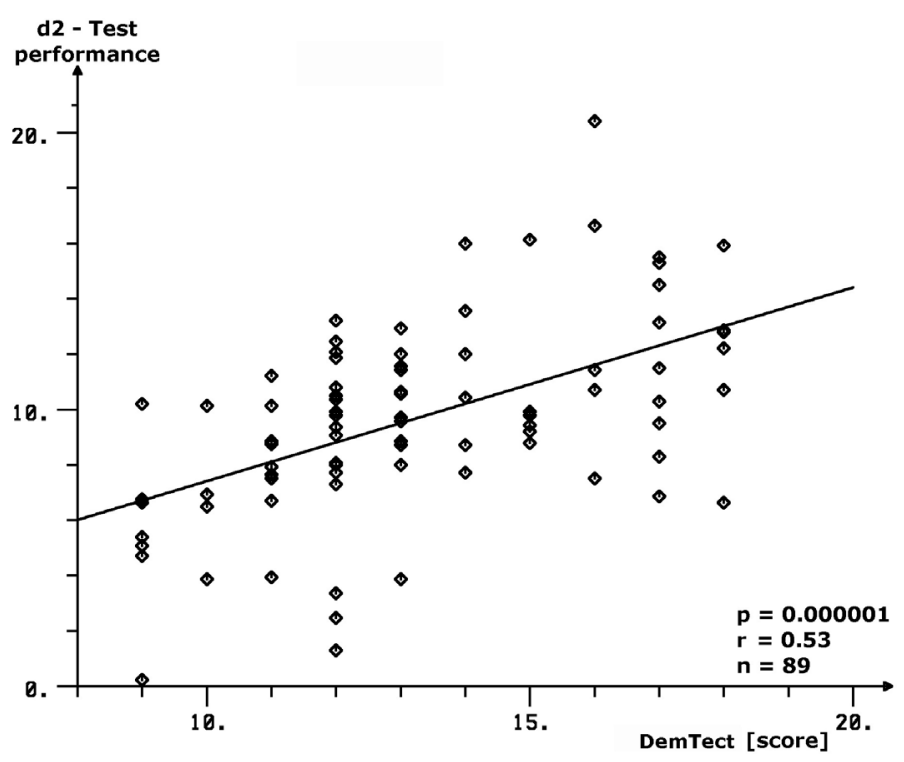

Figure 8. Statistically significant correlation between DemTect score values and psychometric performance in the d2-test. The rank correlation value according to Spearman is given with statistical significance.

\section{Discussion}

Two groups of subjects as defined according to the result of the interactive DemTect questionnaire already differed with respect to absolute voltage in the delta frequency range providing higher values for the MCI group. Largest differences were seen in frontal delta and theta power (electrode positions $F_{7}$ and $F_{8}$ ). This feature of higher delta and theta power has been reported using the same methodology for demented patients in comparison to normal aged matched controls [21]. Other authors also have recently documented higher delta values for subjects suffering from mild cognitive impairment in comparison to healthy volunteers and reported on further alterations typical for those seen in Alzheimer's disease [22]. Higher power in the delta frequency range indicates lower activity of the cholinergic transmitter system [23]. In animal lesion studies, increased delta activity has been reported after destruction of the Nucleus Basalis [24], the main production site of acetylcholine [25]. A comparison of cortical source EEG with MR-based measurements of lobar brain volume (white and grey matter) revealed a negative correlation between the frontal white matter and the amplitude of the delta sources across MCI and Alzheimer subjects (AD), which support a transition hypothesis of brain structural and functional continuity between MCI and AD [26].

Changes of delta and theta waves were also related to pathological changes in cholinergic brain regions. A significant increase of these frequencies was found in patients with the highest total cholinergic burden as well as in patients with highest capsular pathway damage [27]. Abnormal high frontal delta waves under basic relaxed recording conditions can therefore be regarded as indicative for a biochemical and pathological brain dysfunction involving the cholinergic transmitter system.

This also applies to theta waves, which also have been reported to be significantly higher in demented patients than in controls and related to decreased performance in all cognitive domains [28]. In this analysis increase of theta power was observed in most of the brain regions and the difference between the MCI group and the healthy control group (HC) was however not statistically significant with respect to global median values. Very strong evidence for theta power increase as indicator for cognitive decline comes also from longitudinal studies in normal elderly with subjective complaints [1]. With respect to EEG frontal theta power a negative linear correlation was reported to hippocampal volumes for patients suffering from MCI or AD compared to control also indicating fluent transitions for these diseases [29]. These data clearly contradict impressions from nuclear magnetic resonance pictures suggesting only one frontal brain area to be involved in cognitive function. In addition, electric features of brain activity recorded under different performance conditions provide evidence for the involvement of different transmitter activities within different brain regions. There is evidence that theta waves are under the control of the norepinephrine alpha 2 receptor [30]. Also changes of beta frequency ranges have 
been used to successfully predict the clinical status of subjects with MCI over a two years period [31]. However, changes of beta activity did not become statistically significant in my analysis.

Thus, EEG source density measurements in the presence of a relaxed state confirm deviations of electric power within several frequency ranges reported in the literature so far. But like an engine can only be tested under load, brain function should be tested also under "mental" load in order to characterize possible dysfunctions in a more relevant manner. This is possible by recording the EEG in the presence of cognitive performance. Using a battery of different psychometric tests it was recognized long ago that frontal delta and theta power increase under those conditions in healthy volunteers and were related to the difficulty of the task [14]. Interestingly, it was then observed, that demented patients were only able to produce power increases, which were significantly lower than those observed in age matched healthy controls [32]. At the same time a significant correlation was recognized to the severity of the disease as indicated by the interactive questionnaires MMS and ADAS. The present analysis reveals a similar picture. All three psychometric tests induced fronto-temporal increases of delta and theta power in healthy controls. Increases of fronto-central electric theta power have also been observed during other memory demands [33]. Reflection of cognitive and memory performance in the EEG has also been described in detail by others. For example, retrieval of lexical semantic information was linked to theta increases [34]. Furthermore, it was suggested that the encoding of new information is reflected by theta oscillations in hippocampal-cortical feedback loops, whereas search and retrieval processes in (semantic) long-term memory are reflected in upper alpha (alpha 2) oscillations in thalamo-cortical feedback loops [35]. According to animal data alpha 2 frequencies are under the control of dopamine.

In the group of mild cognitive impairment, however, performance of the d2-test led to considerable smaller increases of delta and theta power. Similar results were described for individuals with mild cognitive impairment in the literature [36]. As already recognized in demented patients and confirmed by a correlation analysis between basic theta and event related induced theta [32] this lacking production of fronto-temporal theta waves presumably derives from too high baseline values during the relaxed eyes-open condition. There is obviously a ceiling effect, which prevents further increase of theta power after reaching a physiologically limited maximum. Interestingly, low performance in attention testing was reported to be associated with reduced grey matter density of the left inferior frontal gyrus [37]. In demented patients under the condition of mental load, theta changes were related to the MMS questionnaire. In subjects suffering from mild cognitive impairment a close correlation between theta changes and the score of the DemTect is now observed. This parallel feature speaks in favor of fluent transient states from being healthy via mild cognitive impairment to dementia. But, according to the literature only about every second individual suffering from a decline in cognition develops dementia. In summary, there is compelling evidence, that this change in theta power reactivity can be taken as an indicator for decline of cognition. A longitudinal analysis of future recordings from our subjects will tackle this question.

It can be concluded from our data, that deficits in concentration seem to be the first and most important sign of mild cognitive impairment represented by aberrations in theta activity, followed by already some deficits in memory recognized by deviations in alpha 2 reactivity, whereas arithmetic deficits are not so obvious at this early stage of cognitive impairment. In summary, cognition is a rather complex process, which involves several parts of the brain with increases of electric power in frontal delta and theta waves but also decreases of power in central alpha 2 waves governed by different neurotransmitters. Which of the differences between healthy and mildly impaired subjects are indicative for final development into dementia will hopefully be discovered in future longitudinal studies. But we have now clear neurophysiological parameters to follow in future measurements.

\section{Conclusion}

The present analysis of current source density of the EEG resulted in the detection of quantitative parameters, which are suitable to diagnose mild cognitive impairment at a very early stage. Lower production of theta waves during performance of the d2-test as paper pencil version seems to be the most sensitive neurophysiologic indicator of a cognitive decline. This parameter can now also be used as a non-invasive biomarker for early diagnosis and for testing new drugs aiming at the prevention of development of MCI into dementia.

\section{Competing Interest}

There is no conflict of interest. 


\section{Acknowledgements}

I cordially thank Mrs. Petra Werling, Andrea Cloos and Leonie Schombert for their expert performance and documentation of the results. Mrs. Ingrid K. Keplinger-Dimpfel is appreciated for access of data and quality management. I also thank Prof. Dr. Dr. Hannelore Ehrenreich and Mrs. Dipl. Psychologist Lydia Gibson, Max Planck Institute for Experimental Medicine in Göttingen, Germany, for their critical review.

\section{References}

[1] Prichep, L.S., John, E.R., Ferris, S.H., Rausch, L., Fang, Z., Cancro, R., Torossian, C. and Reisberg, B. (2006) Prediction of Longitudinal Cognitive Decline in Normal Elderly with Subjective Complaints Using Electrophysiological Imaging. Neurobiology of Aging, 27, 471-481. http://dx.doi.org/10.1016/j.neurobiolaging.2005.07.021

[2] Schofield, P.W., Marder, K., Dooneief, G., Jacobs, D.M., Sano, M. and Stern, Y. (1997) Association of Subjective Memory Complaints with Subsequent Decline in Community-Dwelling Elderly Individuals with Baseline Cognitive Impairment. American Journal of Psychiatry, 154, 609-615.

[3] Wang, L., van Belle, G., Crane, P.K., Kukull, W.A., Bowen, J.D., McCormick, W.C. and Larson, E.B. (2004) Subjective Memory Detoriation and Future Dementia in People Aged 65 and Older. Journal of the American Geriatrics Society, 52, 2045-2051. http://dx.doi.org/10.1111/j.1532-5415.2004.52568.x

[4] Treves, T.A., Verchovsky, R., Klimovitzky, S. and Korczyn, A.D. (2005) Incidence of Dementia in Patients with Subjective Memory Complaints. International Psychogeriatrics, 17, 265-273. http://dx.doi.org/10.1017/S1041610205001596

[5] Folstein, M.F., Folstein, S.E. and McHugh, P.R. (1975) Mini-Mental State. A Practical Method for Grading the Cognitive State of Patients for the Clinician. J Psychiatr Res, 12, 189-198.

[6] Kessler, J., Calabrese, P., Kalbe, E. and Berger, F. (2000) DemTect. Ein neues Screening-Verfahren zur Unterstützung der Demenzdiagnostik. Psycho, 26, 343-347.

[7] Kalbe, E., Kessler, J., Calabrese, P., Smith, R., Passmore, A.P., Brand, M. and Bullock, R. (2004) DemTect: A New, Sensitive Cognitive Screening Test to Support the Diagnosis of Mild Cognitive Impairment and Early Dementia. International Journal of Geriatric Psychiatry, 19, 136-143. http://dx.doi.org/10.1002/gps.1042

[8] Düker, H. and Lienert, G.A. (1965) Der Konzentrationsleistungstest (KLT). Göttingen, Hofgrefe.

[9] Jelic, V., Johansson, S.E., Almkvist, O., Shigeta, M., Julin, P., Nordberg, A., Winblad, B. and Wahlund, L.O. (2000) Quantitative Electroencephalography in Mild Cognitive Impairment: Longitudinal Changes and Possible Prediction of Alzheimer's Disease. Neurobiology of Aging, 21, 533-540. http://dx.doi.org/10.1016/S0197-4580(00)00153-6

[10] Alexander, D.M., Arns, M.W., Paul, R.H., Rowe, D.L., Cooper, N., Esser, A.H. and Fallahpour, K. (2006) EEG Markers for Cognitive Decline in Elderly Subjects with Subjective Memory Complaints. Journal of Integrative Neuroscience, 5, 49-74. http://dx.doi.org/10.1016/S0197-4580(00)00153-6

[11] Stomrud, E., Hansson, O., Minthon, L., Blennow, K., Rosen, I. and London, E. (2010) Slowing of EEG Correlates with CSF Biomarkers and Reduced Cognitive Speed in Elderly with Normal Cognition over 4 Years. Neurobiology of Aging, 31, 215-223. http://dx.doi.org/10.1016/j.neurobiolaging.2008.03.025

[12] Harmony, T., Fernandez-Bouzas, A., Marosi, E., Fernandez, T., Bernal, J., Rodriguez, M., Reyes, A., Silva, J., Alonso, M. and Casian, G. (1993) Correlation between Computed Tomography and Voltage and Current Source Density Spectral Parameters in Patients with Brain Lesions. Electroencephalography and Clinical Neurophysiology, 87, 196-205. http://dx.doi.org/10.1016/0013-4694(93)90019-R

[13] Dimpfel, W., Hofmann, H.C., Prohaska, A., Schober, F. and Schellenberg, R. (1996) Source Density Analysis of Functional Topographical EEG: Monitoring of Cognitive Drug Action. European Journal of Medical Research, 1, $283-290$.

[14] Schober, F., Schellenberg, R. and Dimpfel, W. (1995) Reflection of Mental Exercise in the dynamic Quantitative Topographical EEG. Neuropsychobiology, 31, 98-112. http://dx.doi.org/10.1159/000119179

[15] Jasper, H.H. (1958) The Ten-Twenty Electrode System of the International Federation. Electroencephalography and Clinical Neurophysiology, 10, 371-375.

[16] Lehmann, D. (1987) Principles of Spatial Analysis. In: Gevins, A.S. and Remond, A., Eds., Handbook of Electroencephalography and Clinical Neurophysiology, Rev. Series, Vol. 1: Methods of Analysis of Brain and Magnetic Signals, Elsevier, Amsterdam, 309-354.

[17] Dimpfel, W., Kler, A., Kriesl, E., Lehnfeld, R. and Keplinger-Dimpfel, I.K. (2006) Neurophysiological Characterization of a Functionally Active Drink Containing Extracts of Ginkgo and Ginseng by Source Density Analysis of the Human EEG. Nutritional Neuroscience, 9, 213-224. http://dx.doi.org/10.1080/10284150601043713

[18] Berger, H. (1929) Über das Elektroenkephalogramm des Menschen. Archiv für Psychiatrie und Nervenkrankheiten, 87, 527-570. http://dx.doi.org/10.1007/BF01797193 
[19] Brunet, D., Murray, M.M. and Michel, C.M. (2011) Spatiotemporal Analysis of Multichannel EEG: CARTOOL. Computational Intelligence and Neuroscience, 2011, Article ID: 813870. http://dx.doi.org/10.1155/2011/813870

[20] Dimpfel, W., Kler, A., Kriesl, E., Lehnfeld, R. and Keplinger-Dimpfel, I.K. (2007) Source Density Analysis of the Human EEG after Ingestion of a Drink Containing Decaffeinated Extract of Green Tea Enriched with L-Theanine and Theogallin. Nutritional Neuroscience, 10, 169-180. http://dx.doi.org/10.1080/03093640701580610

[21] Schellenberg, R., Todorova, A., Hofmann, H.C., Dimpfel, W. and Schober, F. (1995) Differentiation of Demented Patients and Healthy Subjects by Means of Quantitative-Topological EEG-A Classification Approach. Alzheimer's Research, 1, 23-28.

[22] Babiloni, C., Frisoni, G., Steriade, M., Bresciani, L., Binetti, G., Percio, C.D., Geroldi, C., Miniussi, C., Nobili, F., Rodriguez, G., Zappasodi, F., Carfagna, T. and Rossini, P.M. (2006) Frontal White Matter Volume and Delta EEG Sources Negatively Correlate in Awake Subjects with Mild Cognitive Impairment and Alzheimer's Disease. Clinical Neurophysiology, 117, 1113-1129. http://dx.doi.org/10.1016/j.clinph.2006.01.020

[23] Dimpfel, W. (2005) Pharmacological Modulation of Cholinergic Brain Activity and Its Reflection in Special EEG Frequency Ranges from Various Brain Areas in the Freely Moving Rat (Tele-Stereo-EEG). European Neurospychopharmacology, 15, 673-682. http://dx.doi.org/10.1016/j.euroneuro.2005.03.006

[24] Buzsaki, G., Bickkford, R.G., Ponomareff, G., Thal, L.J., Mandel, R. and Gage, F.H. (1988) Nucleus Basalis and Thalamic Control of Neocortical Activity in the Freely Moving Rat. Journal of Neuroscience, 11, 4007-4026.

[25] Riekkinen Jr., P., Sirviö, J. and Riekkinen, P. (1990) Relationship between the Cortical Choline Acetyltransferase Content and EEG Delta-Power. Neuroscience Research, 8, 12-20. http://dx.doi.org/10.1016/0168-0102(90)90052-g

[26] Babiloni, C., Visser, P.J., Frisoni, G., DeDeyn, P.P., Bresciani, L., Jelic, V., Nagels, G., Rodriguez, G., Rossini, P.M., Vecchio, F., Colombo, D., Verhey, F., Wahlund, L.O. and Nobili, F. (2010) Cortical Sources of Resting EEG Rhythms in Mild Cognitive Impairment and Subjective Memory Complaint. Neurobiology of Aging, 31, 1787-1798. http://dx.doi.org/10.1016/j.neurobiolaging.2008.09.020

[27] Moretti, D.V., Pievani, M., Fracassi, C., Geroldi, C., Calabria, M., De Carli, C.S., Rossini, P.M. and Frisoni, G.B. (2008) Brain Vascular Damage of Cholinergic Pathways and EEG Markers in Mild Cognitive Impairment. Journal of Alzheimer's Disease, 15, 357-372.

[28] Van der Hiele, K., Vein, A.A., Reijntjes, R.H., Westendorp, R.G., Bollen, E.L., van Buchem, M.A., van Dijk, J.G. and Middelkoop, H.A. (2007) EEG Correlates in the Spectrum of Cognitive Decline. Clinical Neurophysiology, 118, 19311939. http://www.ncbi.nlm.nih.gov/pubmed/17604688 http://dx.doi.org/10.1016/j.clinph.2007.05.070

[29] Grunwald, M., Busse, F., Hensel, A., Kruggel, F., Riedel-Heller, S., Wolf, H., Arendt, T. and Gertz, H.J. (2001) Correlation between Cortical Theta Activity and Hippocampal Volumes in Health, Mild Cognitive Impairment, and Mild Dementia. Journal of Clinical Neurophysiology, 18, 178-184. http://www.ncbi.nlm.nih.gov/pubmed/11435810 http://dx.doi.org/10.1097/00004691-200103000-00010

[30] Dimpfel, W. and Schober, F. (2001) Norepinephrine, EEG Theta Waves and Sedation. Brain Pharmacol, 1, 89-97.

[31] Baker, M., Kwaku, A., Schiffer, R. and O’Boyle, M.W. (2008) EEG Patterns in Mild Cognitive Impairment (MCI) Patients. The Open Neuroimaging Journal, 2, 52-55. http://www.ncbi.nlm.nih.gov/pubmed/19018315 http://dx.doi.org/10.2174/1874440000802010052

[32] Schellenberg, R., Todorova, A., Dimpfel, W. and Schober, F. (1997) Pathophysiology and Psycho-Pharmacology of Dementia-A New Study Design. I. Diagnosis Comprising Subjective and Objective Criteria. Neuropsychobiology, 32, 81-97. http://dx.doi.org/10.1159/000119219

[33] Grunwald, M., Weiss, T., Krause, W., Beyer, L., Rost, R., Gutberlet, I. and Gertz, H.J. (1999) Power of Theta Waves in the EEG of Human Subjects Increases during Recall of Haptic Information. Neuroscience Letters, 260, 189-192. http://dx.doi.org/10.1016/S0304-3940(98)00990-2

[34] Bastiaanson, M.C., Oostenveld, R., Jensen, O. and Hagoort, P. (2008) I See What You Mean: Theta Power Increases Are Involved in the Retrieval of Lexical Semantic Information. Brain and Language, 106, 15-28. http://dx.doi.org/10.1016/j.bandl.2007.10.006

[35] Klimesch, W. (1999) EEG Alpha and Theta Oscillations Reflect Cognitive and Memory Performance: A Review and Analysis. Brain Research Reviews, 29, 169-195. http://dx.doi.org/10.1016/S0165-0173(98)00056-3

[36] Deiber, M.P., Ibañez, V., Missonnier, P., Herrmann, F., Fazio-Costa, L., Gold, G. and Giannakopoulos, P. (2009) Abnormal-Induced Theta Activity Supports Early Directed-Attention Network Deficits in Progressive MCI. Neurobiology of Aging, 30, 1444-1452. http://dx.doi.org/10.1016/j.neurobiolaging.2007.11.021

[37] Leyhe, T., Ethofer, T., Bretscher, J., Künle, A., Säuberlich, A.L., Klein, R., Gallwitz, B., Häring, H.U., Fallgatter, A., Klingberg, A., Saur, R. and Müssig, K. (2013) Low Performance in Attention Testing Is Associated with Reduced Grey Matter Density of the Left Inferior Frontal Gyrus in Euthyroid Patients with Hashimoto’s Thyroiditis. Brain, Behavior, and Immunity, 27, 33-37. http://dx.doi.org/10.1016/j.bbi.2012.09.007 